\title{
Tracheal stenosis following mild-to-moderate COVID-19 infection without history of tracheal intubation: a case report
}

\author{
Reza Ershadi $^{1} \cdot$ Shahab Rafieian ${ }^{1} \cdot$ Javad Sarbazzadeh $^{1} \cdot$ Matin Vahedi $^{1}[$
}

Received: 5 October 2021 / Accepted: 14 November 2021 / Published online: 23 November 2021

(c) The Japanese Association for Thoracic Surgery 2021

\begin{abstract}
Laryngotracheal stenosis is a major side effect of intubation in patients with Coronavirus 2019 (COVID-19). However, no study has since reported tracheal stenosis in patients with COVID-19 without a history of tracheal intubation. The current study reports a 77-year-old male patient with the COVID-19 infection but without a history of tracheal intubation diagnosed with distal tracheal stenosis. Tracheal stenosis was successfully treated with rigid bronchoscopy. This study reported the first case of tracheal stenosis due to viral tracheitis associated with COVID-19 infection. However, further studies are required to investigate this speculation.
\end{abstract}

Keywords Tracheal stenosis · Rigid bronchoscopy $\cdot$ COVID-19

\section{Introduction}

Coronavirus 2019 (COVID-19) is an infectious disease that has spread globally and has become a major public health problem [1]. Around 5-12\% of patients with COVID19 require prolonged mechanical ventilation through an endotracheal tube [2]. Laryngotracheal stenosis is a major side effect of intubation in patients with COVID-19 [3]. However, no study has since reported tracheal stenosis in patients with COVID-19 without a history of tracheal intubation. The current study reports a 77-year-old male patient with the COVID-19 infection but without a history of tracheal intubation diagnosed with distal tracheal stenosis. Tracheal stenosis was successfully treated with rigid bronchoscopy. Written informed consent was acquired from the patient for publication of this report.

Reza Ershadi and Shahab Rafieian authors contributed equally to this manuscript.

Matin Vahedi

dr.matinvahedi@gmail.com

1 Department of Thoracic Surgery, Imam Khomeini Hospital Complex, Tehran University of Medical Sciences, Qarib St, Tehran, Iran

\section{Case presentation}

In September 2021, a 77-year-old male patient presented with progressive shortness of breath for the past month and was admitted to the emergency department of Imam Khomeini Hospital, Tehran, Iran. The patient had a previous COVID-19 infection two months earlier, which was presented with mild dyspnea and managed in an outpatient setting. The previous chest computed tomography (CT) scan had shown mild-to-moderate COVID-19 infection with normal upper and lower respiratory tracts (Fig. 1). The previous COVID-19 infection required no admission, intubation, or non-invasive ventilation (NIV). However, after a period of remission, the patient experienced progressive shortness of breath, leading to admission to our department. The past medical history was unremarkable except for diabetes mellitus type 2 (DM-2) (treated with insulin) and an episode of transient ischemic attack (TIA) in the form of hemiparesis several years ago. On physical exam, vital signs were in the normal range, and $\mathrm{O}_{2}$ saturation was $95 \%$. Inspiratory stridor was evident on pulmonary auscultation. Other findings were normal on physical examination.

Laboratory findings were unremarkable, and no abnormal finding was evident in cardiac assessment, including electrocardiogram (ECG) and echocardiography. With suspicion of relapsed COVID-19 infection or infection with mutated strains of COVID-19, a chest CT scan was conducted, surprisingly showing stenosis at the distal part of the trachea 
Fig. 1 Chest CT scan two months earlier showing normal airway lumen

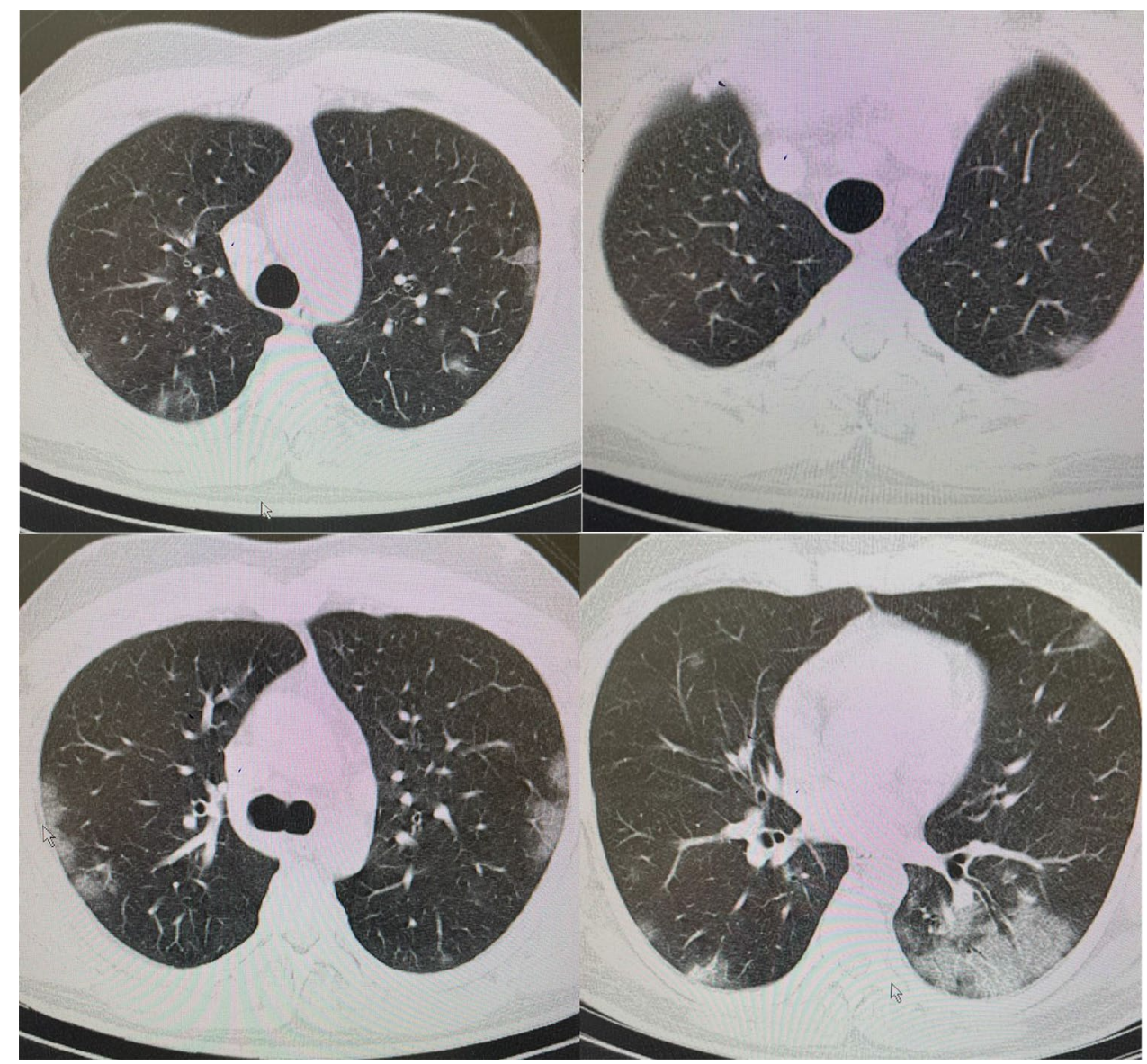

(Fig. 2). The patient underwent flexible bronchoscopy, demonstrating a $25-\mathrm{mm}$-stenosis with a $20 \mathrm{~mm}$ distance from carina and a $70 \mathrm{~mm}$ distance from vocal cords (Figs. 3 and 4). A sample was taken from the tracheal stenosis and sent for pathological assessment that showed mild chronic inflammation with no signs of malignancy or granulomatosis. Further laboratory examination for autoimmune antibodies such as antineutrophil cytoplasmic antibodies (ANCA), rheumatoid factor (RF), and antinuclear antibody (ANA) was negative.

Due to past medical history of DM-2 and old age, the patient was at high risk for definitive surgery. Furthermore, since the exact nature and etiology of the stenosis were not identified, the patient was selected for stage management. The distal tracheal stenosis was dilated with $8.5-\mathrm{mm}$ rigid bronchoscopy leading to significant improvement of dyspnea. In a one-week follow-up, respiratory stridor was absent, and the patient experienced no symptoms. However, rigid bronchoscopy was a temporary treatment for symptom relief, and more follow-ups are undergoing for this patient.

\section{Discussion}

Laryngotracheal stenosis is the partial or complete narrowing of the respiratory tract at the larynx, subglottic space, or trachea. The most common site of tracheal stenosis is the subglottic space, as it is the narrowest part of the normal adult airway [4]. Before the pandemic, laryngotracheal stenosis occurred in up to $9 \%$ of patients with invasive mechanical ventilation. However, during the COVID-19 pandemic, the rate of stenosis after mechanical ventilation has risen because of trauma due to decreased visualization when using personal protective equipment, cuff overinflation, 


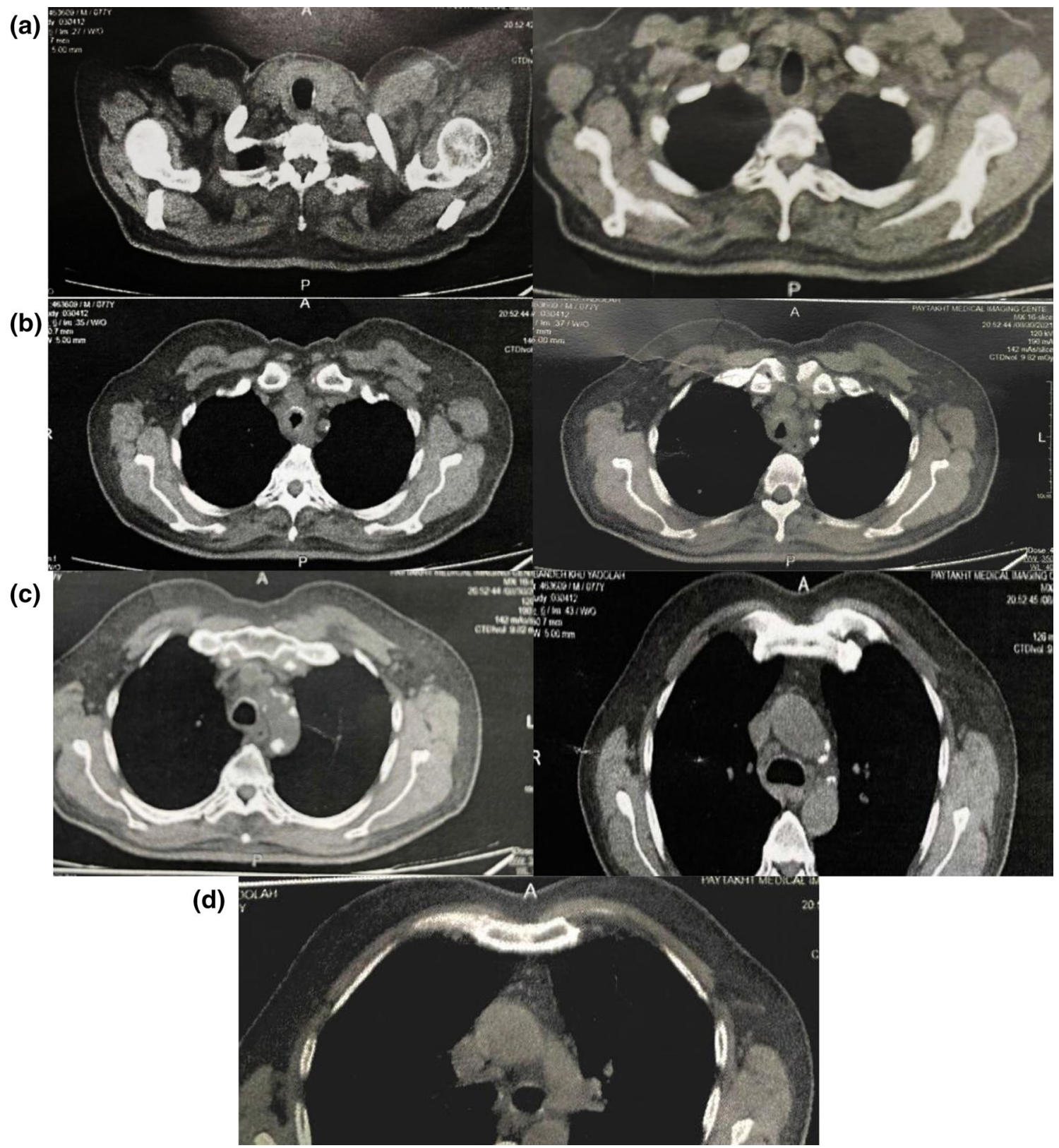

Fig. 2 Current chest CT scan showing stenosis at the distal part of the trachea 


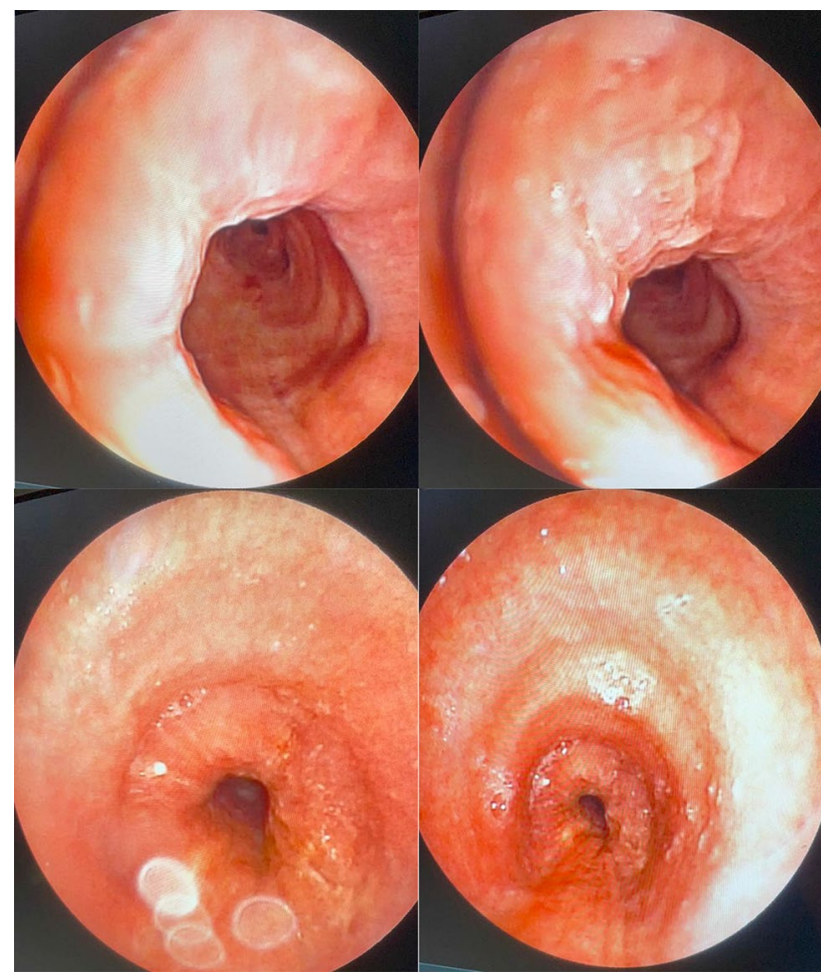

Fig. 3 Images from flexible bronchoscopy demonstrating a narrowing with $80 \%$ obstruction in the distal part of the trachea and prolonged intubation [5]. However, there is no report of tracheal stenosis in patients with COVID-19 that did not require mechanical ventilation. To the best of our knowledge, this is the first report of tracheal stenosis in a patient infected with COVID-19 without a history of endotracheal intubation.

The most critical etiology for tracheal stenosis is trauma secondary to prolonged intubation or excessive endotracheal tube cuff pressure. Nonetheless, other etiologies have been suggested, including infection, gastroesophageal reflux disorder, systemic inflammatory diseases, radiation therapy, primary and secondary tracheal malignancies, and congenital abnormalities [6]. The reported case in this study was an old male patient with normal airway lumen at the beginning of COVID-19 infection with no remarkable past medical history. Moreover, laboratory autoimmune antibodies were negative. Pathologic report also found nonspecific inflammation with no signs of malignancy or granulomatosis-these findings rule out other etiologies except for viral tracheitis due to COVID-19 infection.

\section{Conclusion}

In conclusion, our study is the first report of tracheal stenosis in a patient infected with COVID-19 but without a history of endotracheal intubation, which was successfully treated
Fig. 4 Images from virtual bronchoscopy demonstrating a 25 -mm-stenosis with $20 \mathrm{~mm}$ distance from carina and $70 \mathrm{~mm}$ distance from vocal cords

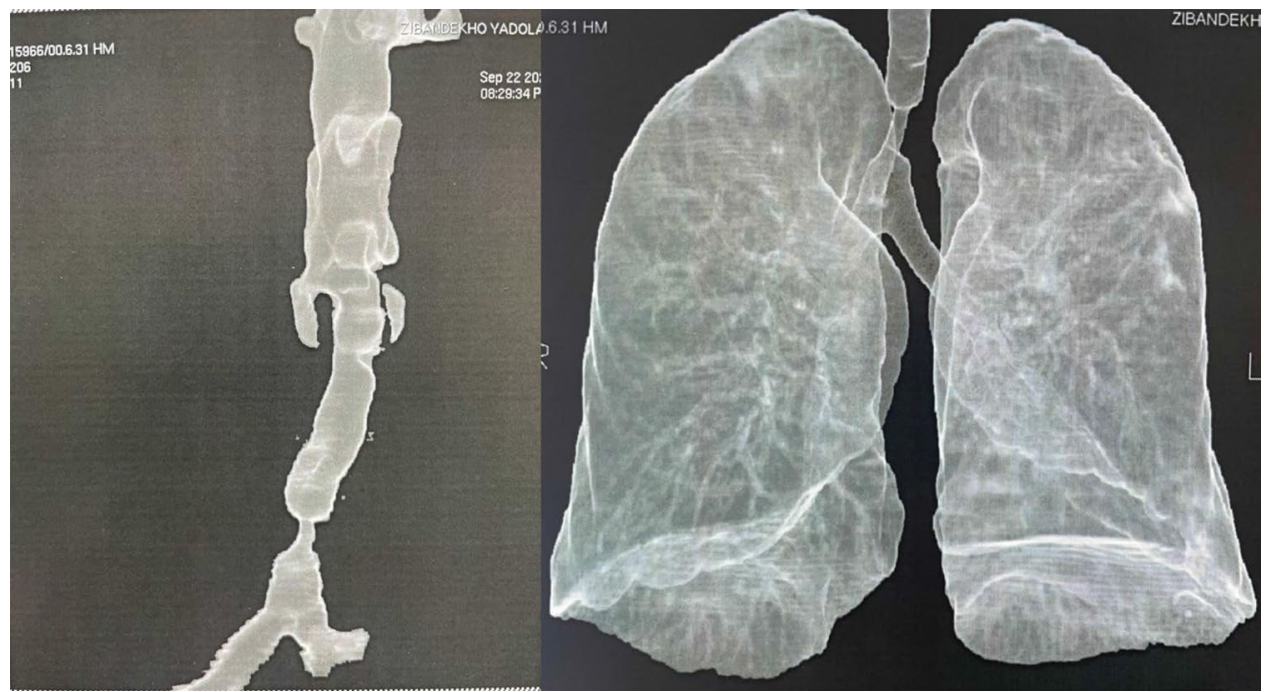


with rigid bronchoscopy. This study reported the first case of tracheal stenosis due to viral tracheitis associated with COVID-19 infection. However, further studies are required to investigate this speculation.

\section{Declarations}

Conflict of interest All authors declare that they have no conflict of interest.

Ethical approval Investigations were in accordance with the Helsinki Declaration of 1964 and all subsequent revisions.

\section{References}

1. Vestergaard LS, et al. Excess all-cause mortality during the COVID-19 pandemic in Europe - preliminary pooled estimates from the EuroMOMO network, March to April 2020. Euro Surveill. 2020;25(26):2001214.

2. Piazza $\mathrm{C}$, et al. Long-term intubation and high rate of tracheostomy in COVID-19 patients might determine an unprecedented increase of airway stenoses: a call to action from the European Laryngological Society. Eur Arch Otorhinolaryngol. 2021;278(1):1-7.

3. Miwa M, et al. Two Cases of Post-intubation Laryngotracheal Stenosis Occurring after Severe COVID-19. Intern Med. 2021;60(3):473-7.

4. Allgood S, et al. Acquired laryngeal and subglottic stenosis following COVID-19-Preparing for the coming deluge. J Clin Nurs. 2021. https://doi.org/10.1111/jocn.15992.

5. Hillel AT, et al. Predictors of posterior glottic stenosis: a multiinstitutional case-control study. Ann Otol Rhinol Laryngol. 2016;125(3):257-63.

6. Aravena $\mathrm{C}$, et al. Idiopathic subglottic stenosis: a review. J Thorac Dis. 2020;12(3):1100-11.

Publisher's Note Springer Nature remains neutral with regard to jurisdictional claims in published maps and institutional affiliations. 